\title{
Search for E(5) Critical Point Symmetry in Light Ge Isotopes
}

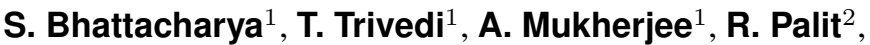 \\ Md.S.R. Laskar ${ }^{2}$, D. Negi ${ }^{2}$, J. Sethi ${ }^{2}$, S. Saha ${ }^{2}$, S. Rajbanshi ${ }^{3}$, \\ S. $\mathrm{Nag}^{4}$, M. Kumar Raju ${ }^{5}$, B.S. Naidu ${ }^{2}$, S. Jadhav ${ }^{2}$, R. Dhonti ${ }^{2}$, \\ A.K. Jain ${ }^{6}$ \\ ${ }^{1}$ Department of Pure \& Applied Physics, Guru Ghasidas Vishwavidyalaya, Koni, \\ Bilaspur-495009, India \\ ${ }^{2}$ Department of Nuclear and Atomic Physics, Tata Institute of Fundamental \\ Research, Mumbai-400005, India \\ ${ }^{3}$ Department of Physics, Presidency University, Kolkata-700073, India \\ ${ }^{4}$ Department of Physics, IIT(BHU), Varanasi-221005, India \\ ${ }^{5}$ Department of Physics, GITAM Institute of Science, Vishakhapatnam-530045, \\ India \\ ${ }^{6}$ Amity Institute of Nuclear Science \& Technology, Amity University UP, \\ Noida-201313, India
}

Received 15 October 2021

doi: https://doi.org/10.55318/bgjp.2021.48.5-6.541

\begin{abstract}
We present here a comparative analysis of even-even $\mathrm{Ge}$ isotopes to further understand the $\gamma$-vibrational bands and $E(5)$ critical point symmetry based on our recent study on ${ }^{68} \mathrm{Ge}$ nucleus [1]. The properties of ${ }^{64-72} \mathrm{Ge}$ isotopes displaying the characteristics of $\gamma$ -band have been discussed in terms of energy systematic and variation of $R(J)_{\gamma, g r}$ parameter. Further, the possibility of the presence of $\mathrm{U}(5)$, $\mathrm{O}(6)$, and $\mathrm{E}(5)$ critical point symmetry in different Ge isotopes are predicted on the basis of experimental level energy ratios of the low lying states. The experimental results of ${ }^{66,68} \mathrm{Ge}$ nucleus are interpreted in terms of interacting boson approximation (IBA) calculations to test the possible existence of $\mathrm{E}(5)$ critical point symmetry.
\end{abstract}

KEY WORDS: $\gamma$-band, E(5) critical point symmetry.

\section{Introduction}

The investigation of atomic nuclei at low excitation energy and angular momenta helps us to improve our understanding of classes of structural symmetries such as harmonic vibrations, deformed gamma-soft, and rigid axially symmetric/triaxial 


\section{S. Bhattacharya et al.}

rotor. For collective nuclei, the last three symmetries can be represented in terms of the algebraic interacting boson model under $\mathrm{U}(5), \mathrm{SU}(3)$, and $\mathrm{O}(6)$ limits. F. Iachello has introduced a new class of dynamic symmetries to describe spectra of a nucleus at or around the critical point of the $\mathrm{U}(5)-\mathrm{SO}(6)$ phase transition [2]. The first-order X(5) critical point symmetry (CPS) is observed in between U(5) and $\mathrm{SU}(3)$ stable symmetries, whereas the second-order $\mathrm{E}(5)$ critical point symmetry lies in between $\mathrm{U}(5)$ and $\mathrm{O}(6)$ symmetries [3]. The second-order $\mathrm{E}(5)$ CPS was first identified in ${ }^{134} \mathrm{Ba}$ nucleus, in which the experimental findings are well interpreted through the IBA calculations. Recently, this has been identified in ${ }^{82} \mathrm{Kr}$ nucleus based on the $\mathrm{B}(\mathrm{E} 2)$ transition strength of low lying states extracted from the lifetime measurement using Doppler-shift attenuation methods [4]. Apart from these nuclei, the E(5) CPS has also been observed in several nuclei such as, ${ }^{102} \mathrm{Pd}[5],{ }^{104} \mathrm{Ru}$ [6], etc. However, observation of this CPS is pretty much limited to the lighter $A \sim 70$ mass region.

The parameters required to comprehend these symmetries include $\beta$ and $\gamma$ deformation parameters. The variation of the $\gamma$ parameter shows a different kind of structural effects, while the $\gamma$-soft region lies between the vibrator and $\gamma$-rigid structure where the potential is $\gamma$ independent. This corresponds to the region between $\mathrm{U}(5)$ and $\mathrm{O}(6)$, where the $\mathrm{E}(5)$ critical point symmetry occurs in the language of the interacting boson approximation (IBA) [7]. In even-even Ge isotopes, the occurrence of $\gamma$-band built on second excited $2^{+}$state has been observed; however, the presence of critical point symmetry E(5) in these nuclei is yet to be explored. In the present work, a comparative study of even-even Ge isotopes has been presented in the context of $\gamma$-vibrational band and $\mathrm{E}(5)$ critical point symmetry. The experimental observations are discussed in terms of interacting boson approximation (IBA) model calculations.

\section{Experimental Data}

We have recently studied the excited states in the ${ }^{68} \mathrm{Ge}$ nucleus through the ${ }^{58} \mathrm{Ni}\left({ }^{16} \mathrm{O}, \alpha 2 \mathrm{p}\right){ }^{68} \mathrm{Ge}$ reaction [1]. A detailed discussion of the experiment is reported in Ref. $[1,8]$. Our study complements the earlier studies on Ge isotopes reported in number of papers [9-12]. All the experimental data used in this paper have been taken from these references and our own work.

\section{$3 \gamma$-Vibrational Band and E(5) Critical Point Symmetry}

The $\gamma$-vibrational bands associated with the ellipsoidal oscillation of nuclei have been observed throughout the nuclear chart. Such excitation causes shape polarization of the nucleus favourable at soft potential energy surfaces for both $\beta$ and $\gamma$ deformation parameters. The presence of $\gamma$-bands have been identified in various even-even ${ }^{64,66,68,70,72} \mathrm{Ge}$ isotopes [1,9-12]. The nature of the $\gamma$-bands can be estimated from $R(J)_{\gamma, g r}$ parameter, defined in terms of the excitation 
Search for E(5) Critical Point Symmetry in Light Ge Isotopes

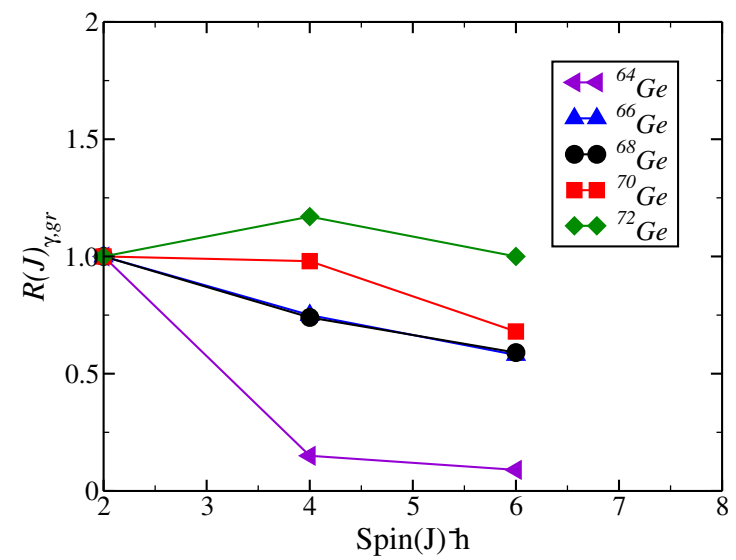

Figure 1. (Color online) The $R(J)_{\gamma, g r}$ as a function of spin $J$ for the quasi- $\gamma$-vibrational bands in ${ }^{64} \mathrm{Ge}[9],{ }^{66} \mathrm{Ge}[10],{ }^{68} \mathrm{Ge}[1],{ }^{70} \mathrm{Ge}[11]$, and ${ }^{72} \mathrm{Ge}$ [12] nuclei.

energies of the ground (gr) state band and $\gamma$-band, [13]

$$
R(J)_{\gamma, g r}=\frac{\left[E\left(J_{\gamma}^{+}\right)-E\left(J_{g r}^{+}\right)\right]}{\left[E\left(2_{\gamma}^{+}\right)-E\left(2_{g r}^{+}\right)\right]} .
$$

$\mathrm{R}(\mathrm{J})_{\gamma, g r}$ is the ratio of the level energies of the $\gamma$ band and the ground state band for a given spin $J$. In a perfect rotor, the inertial parameters for the two bands are the same, and the $R(J)_{\gamma, g r}$ parameter is unity for all $J$ values. The same results are also applicable to the quasi- $\gamma$ band of a harmonic vibrator. The deviation of the value from unity indicates occurrence of other structural effects such as vibrations, gamma-softness, gamma-rigidity.

The variation of $R(J)_{\gamma, g r}$ as a function of spin for different Ge isotopes are shown in Figure 1. A sharp decrease in the value of $R(J)_{\gamma, g r}$ parameter with respect to spin shows the vibrational nature of these bands in ${ }^{64} \mathrm{Ge}$ nucleus. Whereas the increasing value of the $R(J)_{\gamma, g r}$ parameter supports the existence of $\gamma$-rigid nature in ${ }^{72} \mathrm{Ge}$ nucleus. However, in ${ }^{66,68} \mathrm{Ge}$ isotopes, the slowly decreasing trend of $R(J)_{\gamma, g r}$ parameter as a function of spin suggests $\gamma$-soft nature.

Apart from the $\gamma$-soft nature, level energy ratios of low lying states are also important characteristics of E(5) critical point symmetry. Table 1, shows a comparison of various level energy ratios for ${ }^{64,66,68,70,72}$ Ge nuclei with that of ideal $\mathrm{U}(5), \mathrm{O}(6)$ and $\mathrm{E}(5) \mathrm{CPS}$. The values obtained for ${ }^{70,72} \mathrm{Ge}$ nuclei lie close to $\mathrm{U}(5)$ symmetry whereas, for ${ }^{64} \mathrm{Ge}$ nucleus, it is close to $\mathrm{O}(6)$ symmetry. On the other hand, the values observed for the transitional ${ }^{66,68} \mathrm{Ge}$ nuclei suggest they are suitable candidates for E(5) CPS. 


\section{S. Bhattacharya et al.}

Table 1. Comparison of various experimental ratios of ${ }^{64,66,68,70,72} \mathrm{Ge}$ nuclei with the $\mathrm{E}(5), \mathrm{U}(5), \mathrm{O}(6)$ and IBA calculations [1,9-12].

\begin{tabular}{cccccccc}
\hline \hline \multirow{2}{*}{ Symmetry } & $\frac{E\left(4_{1,2}^{+}\right)}{E\left(2_{1,1}^{+}\right)}$ & $\frac{E\left(2_{2,0}^{+}\right)}{E\left(2_{1,1}^{+}\right)}$ & $\frac{E\left(6_{1,3}^{+}\right)}{E\left(2_{1,1}^{+}\right)}$ & $\frac{E\left(8_{1,4}^{+}\right)}{E\left(2_{1,1}^{+}\right)}$ & $\frac{E\left(0_{2,0}^{+}\right)}{E\left(2_{1,1}^{+}\right)}$ & $\frac{E\left(0_{1,3}^{+}\right)}{E\left(2_{1,1}^{+}\right)}$ & $\frac{E\left(0_{2,0}^{+}\right)}{E\left(0_{1,3}^{+}\right)}$ \\
\hline${ }^{64} \mathrm{Ge}$ (expt.) & 2.28 & 1.75 & 3.84 & 5.74 & - & - & - \\
${ }^{66} \mathrm{Ge}$ (expt.) & 2.27 & 1.77 & 3.81 & 5.60 & - & - & - \\
${ }^{68} \mathrm{Ge}$ (expt.) & 2.23 & 1.75 & 3.63 & 4.98 & 3.36 & 3.42 & 1.01 \\
${ }^{70} \mathrm{Ge}$ (expt.) & 2.07 & 1.64 & 3.17 & 4.26 & 2.22 & - & - \\
${ }^{72} \mathrm{Ge}$ (expt.) & 2.07 & 1.76 & 3.32 & 4.51 & 2.43 & 3.30 & 1.36 \\
$\mathrm{E}(5)(\mathrm{N}=\infty)$ & 2.20 & 2.20 & 3.59 & 5.17 & 3.03 & 3.61 & 1.19 \\
$\mathrm{U}(5)$ & 2.00 & 2.00 & 3.00 & 4.00 & 2.00 & 3.00 & 1.50 \\
$\mathrm{O}(6)$ & 2.50 & 2.50 & 4.50 & 7.00 & $>7.00$ & - & - \\
\hline \hline
\end{tabular}
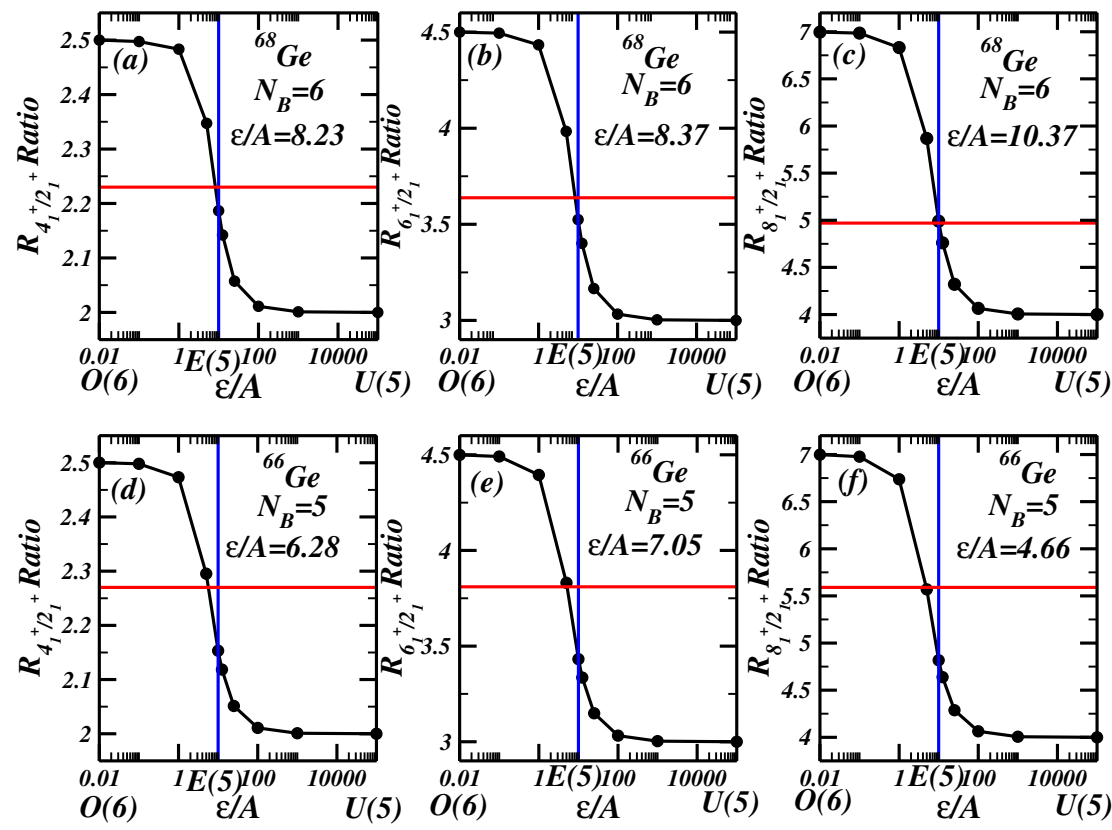

Figure 2. (Color online) Variation of key observables:(a) $R_{4_{1,2}^{+} / 2_{1,1}^{+}}$for $N_{B}=6$; (b) $R_{6_{1,3}^{+} / 2_{1,1}^{+}}$for $N_{B}=6$; (c) $R_{8_{1,4}^{+} / 2_{1,1}^{+}}$for $N_{B}=6$; (d) $R_{4_{1,2}^{+} / 2_{1,1}^{+}}$for $N_{B}=5$; (e) $R_{6_{1,3}^{+} / 2_{1,1}^{+}}$for $N_{B}=5$; (f) $R_{8_{1,4}^{+} / 2_{1,1}^{+}}$for $N_{B}=5$, represented by black solid curves, from $\mathrm{U}(5)$, through the $\mathrm{E}(5)$ (denoted by a vertical solid blue line), to $\mathrm{O}(6)$ against parameter $\epsilon / A$ of IBA Hamiltonian (see text for details). The experimental values are indicated by red solid line. 


\section{Interacting Boson Approximation (IBA) Calculations}

The existence of critical point E(5) symmetry can be explored from the IBA model calculations [14] using the following Hamiltonian:

$$
H=\epsilon n_{d}+A P^{\dagger} P
$$

where $\epsilon, \mathrm{n}_{d}$, and $A$ are the unperturbed energy of a state, number of noninteracting d-bosons, and energy due to pairing interaction, respectively. At the critical point $\mathrm{E}(5)$ symmetry, the value of $\epsilon / A\left[=2\left(N_{B}-1\right)\right]$ ratio is 10 for $N_{B}=6$ and $\epsilon / A=8$ for $N_{B}=5$. The energy levels for $\mathrm{U}(5)$ spherical vibrator and $\mathrm{O}(6) \gamma$-soft structure have been described by the quantum numbers $N_{B}$ and $\sigma$, respectively.

In the present work, the variation of six energy ratios $\left[R_{4_{1,2}^{+} / 2_{1,1}^{+}}\left(\equiv E_{4^{+} / 2^{+}}\right)\right.$, $R_{6_{1,3}^{+} / 2_{1,1}^{+}}\left(\equiv E_{6^{+} / 2^{+}}\right), R_{8_{1,4}^{+} / 2_{1,1}^{+}}\left(\equiv E_{8^{+} / 2^{+}}\right), R_{0_{2,0}^{+} / 2_{1,1}^{+}}\left(\equiv E_{0_{x i}^{+} / 2^{+}}\right)$, $R_{0_{1,3}^{+} / 2_{1,1}^{+}}\left(\equiv E_{4 \tau / 2^{+}}\right)$, and $R_{0_{1,3}^{+} / 0_{2,0}^{+}}\left(\equiv E_{0_{\tau}^{+} / 0_{\xi}^{+}}\right)$, etc.) with respect to the $\epsilon / A$ parameter are compared with the experimental data as shown in Figure 2. The experimental results of ${ }^{68} \mathrm{Ge}$ nucleus shows that the average value of the $\epsilon / A$ is $\approx 10.01$, whereas the average $\epsilon / A$ value is 6.00 for ${ }^{66} \mathrm{Ge}$ nucleus. Although, the $\epsilon / A$ value for ${ }^{66} \mathrm{Ge}$ has been obtained only from the energy ratios of yrast band. These results suggest that the ${ }^{68} \mathrm{Ge}$ nucleus is more favorable candidate than ${ }^{66} \mathrm{Ge}$ nucleus for $\mathrm{E}(5)$ critical point symmetry.

\section{Summary}

The present work reports a systematic comparison of structural behaviour, in the context of $\gamma$-vibration and $\mathrm{E}(5)$ symmetry, for different even-even Ge isotopes. The $\gamma$-vibration, $\gamma$-soft and $\gamma$-rigid nature of ${ }^{64} \mathrm{Ge},{ }^{66,68} \mathrm{Ge}$ and ${ }^{72} \mathrm{Ge}$ isotopes, respectively has been studied from the variation of $R(J)_{\gamma, g r}$ parameter as a function of spin. Further, the typical values of the energy ratios for different even-even Ge isotopes predict the possible existence of E(5) CPS in ${ }^{66,68} \mathrm{Ge}$ isotopes. The agreement between the experimental data and theoretical prediction of IBA calculations suggests that the ${ }^{68} \mathrm{Ge}$ nucleus is a good candidate for $E(5)$ critical point symmetry.

\section{Acknowledgments}

The authors acknowledge the INGA collaborations for establishing the clover array. This work has been partially funded by the Department of Science and Technology, Government of India (No. IR/S2/PF-03/2003-II). Thanks to the TIFR pelletron staff for the smooth functioning of the accelerator. S. Bhattacharya would like to acknowledge the financial assistance from the UGC-DAE-CSR project (UGC-DAE-CSR-KC/CRS/19/NP04/0915) and the IUAC project (IUAC 


\section{S. Bhattacharya et al.}

/XIII.3A/UFR-55313). A.K.J acknowledges the support from SERB (Govt. of India) in the form of grant CRG/2020/000770.

\section{References}

[1] S. Bhattacharya et al. Evidence for shape coexistence and E(5) symmetry in ${ }^{68} \mathrm{Ge}$ nucleus (under review).

[2] F. Iachello (2000) Dynamic Symmetries at the Critical Point. Phys. Rev. Lett. 85 3580.

[3] R.F. Casten and N.V. Zamfir (2000) Evidence for a possible E(5) symmetry in ${ }^{134}$ Ba. Phys. Rev. Lett. 853584.

[4] S. Rajbanshi et al. (2021) Experimental evidence of exact E(5) symmetry in ${ }^{82} \mathrm{Kr}$. Phys. Rev. C 104 L031302.

[5] N.V. Zamfir et al. (2002) ${ }^{102} \mathrm{Pd}$ : An E(5) nucleus? Phys. Rev. C 65044325.

[6] A. Frank, C.E. Alonso, and J.M. Arias (2001) Search for E(5) symmetry in nuclei: The Ru isotopes. Phys. Rev. C 65014301.

[7] E.A. McCutchan, D. Bonatsos, N.V. Zamfir, and R.F. Casten (2007) Staggering in $\gamma$-band energies and the transition between different structural symmetries in nuclei. Phys. Rev. C 76024306.

[8] R. Palit et al. (2012) A high speed digital data acquisition system for the Indian National Gamma Array at Tata Institute of Fundamental Research. Nucl. Instrum. Meth. A 68090.

[9] P.J. Ennis et al. (1991) Triaxiality and isospin-forbidden $E 1$ decays in the $N=Z$ nucleus ${ }^{64}$ Ge. Nucl. Phys. A 535392.

[10] E.A. Stefanova et al. (2003) Four-quasiparticle alignments in ${ }^{66}$ Ge. Phys. Rev. $C 67$ 054319.

[11] R.A. Haring-Kaye et al. (2018) Multiple band structures in ${ }^{70}$ Ge. Phys. Rev. C 97 024308.

[12] D.G. Roux et al. (2012) In-beam spectroscopy of ${ }^{72}$ Ge. Eur. Phys. J. A 4899.

[13] R.F. Casten, R.B. Cakirli, D. Bonatsos, and K. Blaum (2020) Simple new signature of structure in deformed nuclei: Distinguishing the nature of axial asymmetry. Phys. Rev. C 102054310.

[14] O. Scholten (1979) The program package PHINT for IBA calculations. Kernfisisch Versneller Instituut Internal Report No. 63. 